\title{
Systematic Preprocessing of Data Dependent Constructs for Embedded Systems
}

\author{
Martin Palkovic, ${ }^{1, *}$ Erik Brockmeyer, ${ }^{1}$ Peter Vanbroekhoven, ${ }^{2}$ \\ Henk Corporaal, ${ }^{3}$ and Francky Catthoor ${ }^{1}$ \\ 1 IMEC Laboratory, Kapeldreef 75, 3001 Leuven, Belgium \\ ${ }^{2}$ KU Leuven, Celestijnenlaan 200A, 3001 Leuven, Belgium \\ ${ }^{3}$ TU Eindhoven, Den Dolech 2,5612 AZ Eindhoven, The Netherlands
}

(Received: 14 December 2005; Accepted: 23 January 2006)

\begin{abstract}
Data transfers and storage are dominating contributors to the area and power consumption for all modern multimedia applications. A cost-efficient realisation of these systems can be obtained by using high-level memory optimisations. This paper demonstrates that the state-of-the-art memory optimisation techniques only partly can deal with code from real-life multimedia applications. We propose a systematic preprocessing methodology that can be applied on top of the existing work. This opens more opportunities for existing memory optimisation techniques. Our methodology is complemented with a postprocessing step, which eliminates the negative effects of preprocessing and may further improve the code quality. ${ }^{13,18}$ Our methodology has been applied on several reallife multimedia applications. Results show a decrease in the number of main memory accesses up to $45.8 \%$ compared to applying only state-of-the-art techniques.
\end{abstract}

Keywords: Source Code Preprocessing, Data Dependent Constructs, High-Level Transformations, Global Loop Transformations, Profiling, Scenarios, MP3 Audio Decoder.

\section{INTRODUCTION}

Modern multimedia systems are characterised as applications with huge amount of data transfers and large memories. Thus, the memory subsystem in these applications consumes a major part of the overall area and energy. The utilisation of the memory subsystem by optimisation of global memory accesses usually brings significant energy savings. ${ }^{8}$ Improving the temporal locality of the memory accesses also decreases the life-time of data elements and hence the required memory footprint.

Every recent high-level low-power design methodology contains a global loop transformation $(G L T)$ stage. ${ }^{8,20,26}$ This stage either improves the parallelization opportunities at the instruction or data level, or it improves the locality of data accesses so that data can be stored in lower levels of the memory hierarchy, resulting in significant area and power gains.

The loop transformations are nearly always performed on a geometrical model ${ }^{32-36}$ which is very effective in dealing with generic loop transformations. ${ }^{11,20,26,37}$ However,

\footnotetext{
${ }^{*}$ Author to whom correspondence should be addressed.

Email: palkovic@imec.be
}

the geometrical model imposes strict limitations on the input code, namely it considers only the static control parts (SCoP). ${ }^{6}$ The static control part is a maximal set of consecutive statements without while loops, where loop bounds and conditionals may only depend on invariants within this set of statements. These invariants include symbolic constants, formal function parameters and surrounding loop counters. Also, the geometrical model requires pointer-free single-assignment code in one function. ${ }^{8}$ Clearly, this is not acceptable for the initial source code from a user and application point of view.

To alleviate this situation we propose a systematic preprocessing methodology dealing with data dependent constructs present in the initial source code which are the major limitation for geometrical model. In this paper, we do not consider applications with dynamic data types and dynamic memory allocation because this is out of the scope of the GLT and high-level optimizations we are using as a backend. For effective dynamic memory management methods, see Ref. [4]. The novel steps of our methodology addressed in this paper, are applied on top of the earlier published work in preprocessing (see Section 2) as shown in Figure 1. All of our steps described in this paper are performed manually, but in a fully systematic 


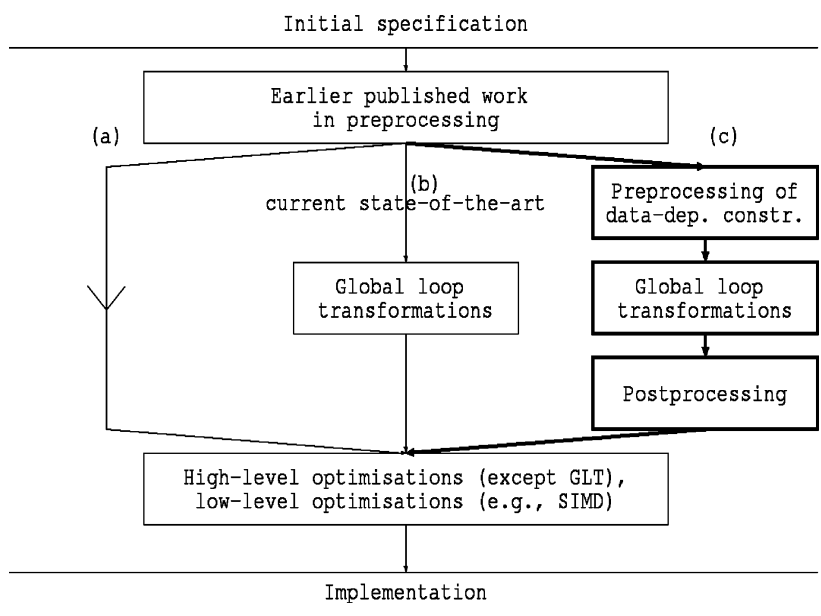

Fig. 1. Optimisation approaches compared in the paper: (a) Without GLT, (b) with GLT and existing preprocessing, (c) with GLT and our systematic preprocessing on top of existing design flow.

way. The automation process is ongoing and it is out of the scope of the paper. For details about the current automation status see Refs. [29, 30].

Our methodology enlarges the exploration scope of the GLT by creating scenarios based on similarity measure and moving the data dependent conditions down in the loop hierarchy. We demonstrate these principles on a simple motivation example of a decoder that decodes three types of frames, P, B, and I frames (see Fig. 2a). The code for decoding the $\mathrm{P}$ frame is similar to the code for decoding the B frame, i.e., a lot of the same decoding moduleskernels (like motion compensation) is used for both, $\mathrm{P}$ and $\mathrm{B}$ frames. The I frame uses a lot of completely different modules-kernels for decoding. Thus, based on the similarity measure, it is beneficial to group the decoding of $\mathrm{P}$ and $\mathrm{B}$ frames together (first scenario) and keep decoding of

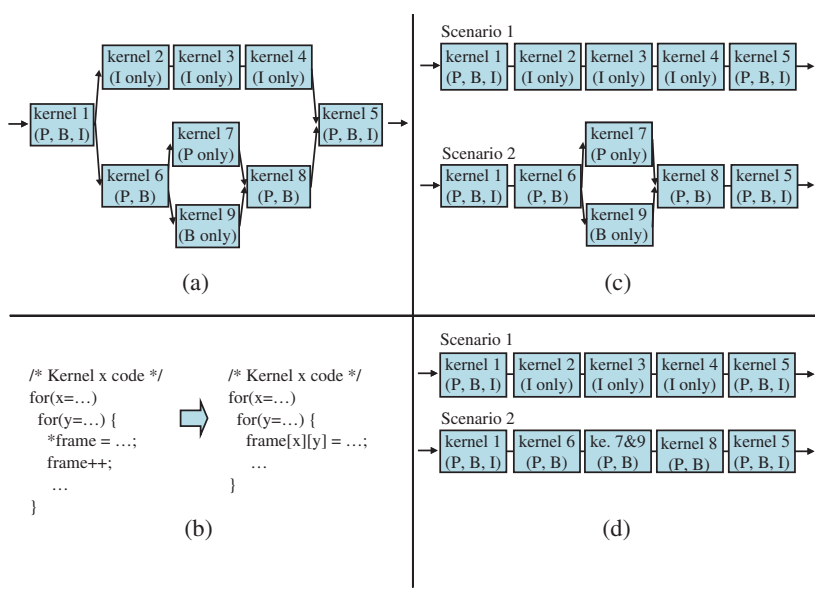

Fig. 2. The state of the art preprocessing focuses only on the individual kernels: (a) Block diagram of the application. (b) Local view of the state of the art preprocessing - the state of the art in preprocessing does not change anything in the block diagram. (c) Scenario creation and (d) moving data dependent conditions down in the loop hierarchy changes the block diagram.
I frames separate (second scenario) as shown in Figure 2c. In the scenario code which decodes I frames no data dependent constructs are present, it is just the pure sequential processing of the I frame. In the scenario code which decodes $\mathrm{P}$ and $\mathrm{B}$ frames data dependent conditions are still present which decide on the particular module-kernel for $\mathrm{P}$ or B frame. These constructs are hidden outside the scope of the GLT by the second part of our methodology which moves non-innermost data dependent conditions down in the loop hierarchy (see Fig. 2d). Together with other existing steps (see Fig. 2b and Section 2) such preprocessed applications can exploit the benefits of the geometrical model and global loop transformations what was not feasible for the whole application before applying our preprocessing methodology.

The rest of the paper is structured as follows. Section 2 surveys related work in global loop transformations and preprocessing. In Section 3 we describe MP3 audio decoder which is used as an example in this paper. Section 4 explains our preprocessing methodology. Section 5 describes our results on four real-life multimedia benchmarks and compares the results to the state-of-the-art work. Finally, in Section 6 we draw several conclusions.

\section{RELATED WORK}

Initial research in loop transformation for locality has focused on optimising locality in single perfectly nested loop by applying a single loop transformation. ${ }^{37}$ This approach did not allow to optimise large buffers necessary for keeping data between loop nests. In Ref. [20] the perfectly nested loop nests are not required, but it is reported that the algorithm does not obtain very good results for imperfectly nested loop nests. The more generic approach for loop transformations involves applying a loop transformation on every statement in the loop nest. ${ }^{11,14}$ This research was mainly focused on parallelisation, through. Moreover the approaches remain rather theoretical with scaling problems when dealing with practical applications where dozens of loop nests with lots of arrays can be present. McKinley et al. in Ref. [26] have improved data locality based on a simple yet accurate model which considers both temporal and spatial locality. However, only a limited set of loop transformations were driven by this model. Another method to improve data locality is to perform data transformations ${ }^{9}$ or use fine-grain scheduling techniques. ${ }^{3}$

Most of the research in this field was based on the use of geometrical models where the set of transformations is limited to affine matrices. ${ }^{10-12,14,15,21,24,25}$ Other researchers did not use a geometrical model at all. Thus, they can usually apply only a very limited set of linear transformations. . $^{2,28,31}$

The state-of-the art in application preprocessing which overcomes the strict limitations of the geometrical 
model used by most loop transformation approaches includes selective function inlining ${ }^{1}$ (SFI), pointer analysis and conversion ${ }^{33,16}$ (PA\&C), dynamic single assignment (DSA) conversion, ${ }^{35}$ and hierarchical rewriting. ${ }^{8}$ Hierarchical rewriting partitions an application in three function layers. The first layer contains process control flow, the second layer contains loop hierarchy and indexed signals, and the third layer contains arithmetic, logic and datadependent operations (see also the Fig. 4a). Only the second layer containing memory management is the target for high-level low-power optimisation.

As mentioned in the introduction, loop transformations performed on geometrical model do not deal with data dependent behaviour. 6 Still, some related work can be found in the high level hardware synthesis area. To deal with data-dependent control flow changes, a transformation technique to convert an iterative algorithm whose loop bounds are data dependent to an equivalent data independent regular algorithm has been proposed in Ref. [19]. However, the authors use the worst case assumption to define the structure of a given situation and lose information about data-dependent iterator bounds. In Ref. [22] a special internal representation is used to treat both dataflow and control-flow designs. This representation is meant for developing scheduling heuristics and it does not enable the GLT.

\section{BENCHMARK DESCRIPTION AND PROFILING}

We use one of our applications, the MP3 decoder, ${ }^{22}$ as example during the rest of the paper. The MP3 decoder is a frame-based algorithm for decoding a bitstream from the perceptual audio coder. A frame is coding 1152 mono or stereo samples and is divided into two granules of 576 samples. Each granule consists of 32 subband blocks of 18 frequency lines and has two channels. One channel in one granule is called a block.

After receiving a frame, the frame is Huffman decoded (see decoder frontend in Fig. 3). Then, on the Huffman

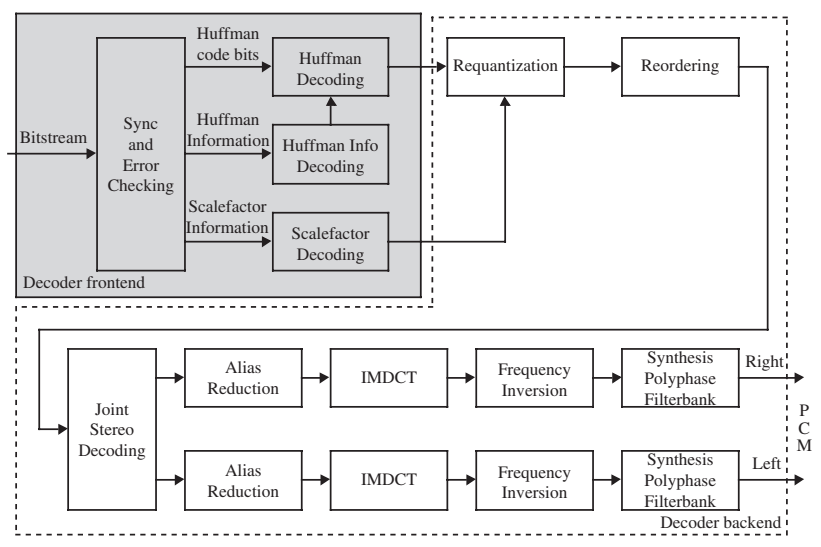

Fig. 3. MP3 audio decoder structure.
Table I. Frequency of use (in \%) of different modes in MP3 audio decoder for real-life bitstreams. The information will be used in Section 4, Subsection 4.1.

\begin{tabular}{lcc}
\hline Mode & Frequency (\%) & Scenario \\
\hline $\begin{array}{l}\text { Long block (type 0)/Joint middle } \\
\quad \text { side stereo decoding }\end{array}$ & 90.1 & 1 \\
$\quad \begin{array}{l}\text { Long block (type 0)/Stereo decoding } \\
\text { Long block (type 1\&3)/Joint middle }\end{array}$ & 3.2 & 1 \\
$\quad$ side stereo decoding & 3.1 & 1 \\
$\quad \begin{array}{l}\text { Long block (type 1\&3)/Stereo decoding } \\
\text { Short block (type 2)/Joint middle }\end{array}$ & 0.9 & 1 \\
$\quad$ side stereo decoding & 1.4 & 2 \\
$\quad \begin{array}{l}\text { Short block (type 2)/Stereo decoding } \\
\quad \text { other }\end{array}$ & 1.2 & 2 \\
& $<0.1$ & 3 \\
\hline
\end{tabular}

decoded frame, several kernels are applied (see the decoder backend in Fig. 3). For details about the different kernels, see Ref. [22]. In the rest of the paper, we will concentrate only on the decoder backend. The decoder frontend (Huffman decoder) is not data access intensive compared to the rest of the whole decoder and is also not suited for highlevel memory optimization because of irregular memory accesses and a lot of small look-up tables.

For the decoding of the MP3 blocks (a block is one channel in one granule), different methods are used depending of the initial coding of the block on the encoder site. Each block can be coded using short window, mixed window or one of three types of long window. Also, each block can be coded using stereo decoding or joint stereo decoding (middle side and/or intensity stereo decoding). Combination of these options introduces different modes of decoding, which are represented with data dependent conditions in the benchmark code. However, the occurrence of different modes is not the same. To identify the most probable ones, we have profiled our application with dozens realistic Mp3 bitstreams (see Table I).

\section{SYSTEMATIC PREPROCESSING OF DATA DEPENDENT CONSTRUCTS}

We consider that the state of the art preprocessing steps listed in Section 2 have already been completed on the initial source code before applying the remaining steps of our methodology. In particular, we have applied SFI, PA\&C, DSA, and hierarchical rewriting. In the MP3 audio decoder, one kernel was unrolled by a factor of 2 . We have decided to roll-up this kernel which improved the structure of the code and allowed later better opportunities for GLT (loop fusion).

An example of the code after preprocessing is shown in Figure 4a. Layer 1 contains process control flow, i.e., reading, decoding, and writing out one frame (1152 stereo samples). Layer 2 describes the loop hierarchy and indexed array signals of the decoding itself. This layer contains all information that is relevant for memory management which is the target for the high-level 


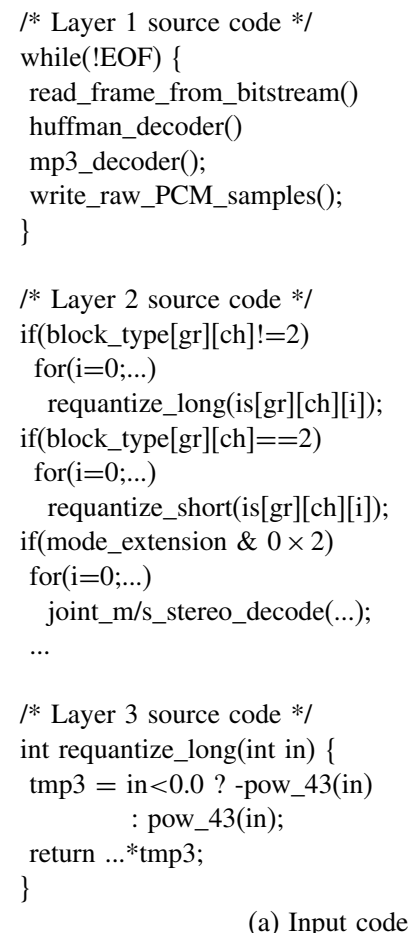

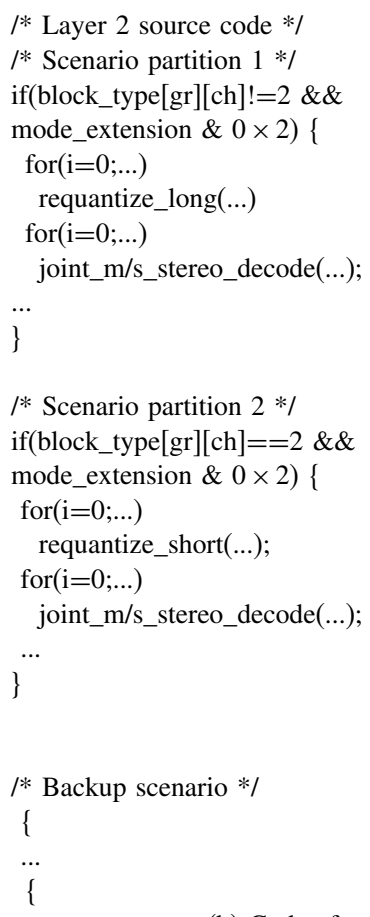

(b) Code after step 4.1

Fig. 4. (a) MP3 code after hierarchical rewriting. (b) Scenarios for an MP3 decoder.

low-power optimisation. Layer 3 contains arithmetic, logic (e.g., requantization of one sample in the long block) and data-dependent operations (so this already eliminates the innermost conditions from the layer 2 code). The second layer is the input code for three new preprocessing steps addressed in this paper, namely:

(1) Identifying scenarios from profiling information

(2) Moving non-innermost data dependent conditions to layer 3

(3) Extending data dependent iterator bounds

In the sequel all three new steps will be explained and demonstrated on different parts of the MP3 decoder.

\subsection{Identifying Scenarios from Profiling Information}

In real-life multimedia applications, various parts of the bitstream are coded with different methods depending on audio and video characteristics of the input signal (see Section 3 for an MP3 audio decoder). These different options introduce different modes of decoding as we discussed in previous Section (see Table I), with possibly different computation and memory requirements. A set of modes is called a scenario. Scenarios are interesting because they can remove the outermost data dependent if conditions which are preventing from performing the GLT.

Identifying scenarios means first collecting the profiling information of all possible modes. Similar modes are then grouped together and create a scenario. Similarity is measured as ratio of the number of identical statements covered by execution of two decoding modes and the total number of statements covered by these modes, i.e.,

$$
\begin{aligned}
& \text { Similarity }\left(\operatorname{mode}_{i}, \operatorname{mode}_{j}\right) \\
& =\frac{\| \text { statements }\left(\operatorname{mode}_{i}\right) \cap \text { statements }\left(\operatorname{mode}_{j}\right) \|}{\| \text { statements }\left(\operatorname{mode}_{i}\right) \cup \text { statements }\left(\operatorname{mode}_{j}\right) \|}
\end{aligned}
$$

Note, that if $i=j$ all statements are identical (we compute the similarity of the same mode) and the Similarity is 1 . When there are no common statements in mode ${ }_{i}$ and mode $_{j}$ the Similarity is 0 . The similarity is computed only for modes, that are relevant, i.e., first $n$ most frequently used modes that cover $90 \%$ of the whole execution time of the application. For the MP3 we have observed that only 6 modes cover $99.9 \%$ of the whole execution time so we took all of them. The 6 modes cover approximately $26 \%$ of all possible modes.

After profiling and computing the similarity for the $n=6$ most frequently used modes we have grouped together modes that had similarity measure close to 1 . Using this approach we have created 2 scenarios for the MP3 audio decoder. The first scenario groups long blocks type $0,1,3$ coded with stereo or middle side coding (see Table I). The second scenario contains short blocks type 2 coded with stereo or middle side coding. For the rest, i.e. the 18 uncovered modes we create one backup scenario.

By creating scenarios, we eliminate most of the outermost data dependent conditions in the code targeted for optimisation. This is done by separation of modes to different groups we call scenarios (compare layer 2 in 
Fig. 4a and Fig. 4b). On the other hand, this leads to significant code size overhead due to the duplication of the code. The cost of scenario creation is mainly in code duplication and thus in the code size increase. The gain is in the reduction of number of memory accesses to expensive off-chip memories after high-level GLT. These transformations can be also beneficial for code size as we will see in the sequel. Without scenario creation these transformations will not be feasible. However, scenarios need to be detected effectively at the run time in the modified code.

To illustrate this on our benchmark, with the grouping mentioned above, we have initially increased the code size by $50 \%$. However, this grouping has enabled GLT that have not been feasible before. After these transformations the overall code size increase is only $2 \%$, while the reduction in the number of main data memory accesses is significant as we will see in Section 5. In general, we group paths with the highest similarity measure, thus the code size increase is kept under control. The remaining steps of the methodology do not increase the code size at all.

\subsection{Moving Non-Innermost Data Dependent Conditions to Layer 3}

In this step we move data dependent if conditions down in the loop hierarchy.

We have eliminated most of the outermost data dependent conditions by creating scenarios as we have described in Subsection 4.1. However, one scenario contains several modes, i.e. some outermost data dependent conditions remain. Example, in scenario 1 of our MP3 audio decoder example (long blocks type 0,1,3 and stereo/joint middle side stereo decoding) the data dependent condition in stereo decoding kernel is present (see Fig. 5a). Note, that the data dependent condition which decides the active one among long blocks $0,1,3$ is innermost and is already hidden in layer 3 of the IMDCT kernel. Also, the code can contain data dependent conditions in the middle of the loop hierarchy.

To eliminate the rest of the outermost and middle data dependent conditions we move them down in the loop hierarchy to hide them also in layer 3 . When moving down in the loop hierarchy, the condition is evaluated many more times. If these conditions create too much overhead for the control-flow, they can be optimized using techniques similar to Falk et al. ${ }^{13}$ after GLT. Also, for our example, the stereo decoding kernel is the only place where the data

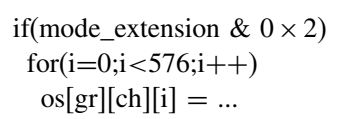

(a)

Fig. 5. Stereo kernel before (a) /after (b) moving data dependent condition to layer 3 .

$$
\begin{aligned}
& \text { for }(\mathrm{i}=0 ; \mathrm{i}<\operatorname{count} 1[\mathrm{gr}][\mathrm{ch}] ; \mathrm{i}++) \\
& \ldots=\text { requantize_long }(\ldots)
\end{aligned}
$$

(a)

$$
\begin{aligned}
& \text { for }(\mathrm{i}=0 ; \mathrm{i}<576 ; \mathrm{i}++) \\
& \text { if }(\mathrm{i}<\text { count } 1[\mathrm{gr}][\mathrm{ch}]) \\
& \ldots=\text { requantize_long }(\ldots) ;
\end{aligned}
$$

(b)
Fig. 6. Requantization kernel before (a) /after (b) data dependent iterator bounds extension.

dependent condition is preventing the loop fusion of all kernels. So to hide his condition in layer 3 may have a huge positive impact on data transfers to main memory and thus on overall power consumption. This stresses the importance of moving the condition down.

Note, that the step 4.2 is complementary with the step 4.1 and together they solve the whole problem of data dependent conditions in the application. That is, the outermost and middle data dependent conditions that have not been handled in step 4.1 are moved to layer 3 in step 4.2. The overhead of moving data-dependent conditions to layer 3 will be eliminated in our postprocessing step by applying the hoisting techniques of Gupta et al. ${ }^{18}$ and the loop nest splitting techniques by Falk et al. ${ }^{13}$

\subsection{Extending Data Dependent Iterator Bounds}

In this step we replace the data dependent iterator bound by a worst case bound and we add a data dependent if condition inside the loop.

In the MP3 decoder the decoded data are divided in three regions: big_value, count1 and rzero region. The borders of the count 1 region are data dependent and only first two regions are decoded in the requantization kernel. This is resulting in a data dependent iteration bound (see Fig. 6a) which is not acceptable for GLT. Thus, we have to extend the data dependent iterator bound to the worst case and data dependent condition inside the loop (see Fig. 6b). The extension to worst case increases the number of iterations of that particular loop, however could be beneficial for energy after applying GLT.

If we use the worst case for the upper bound (576 samples) in the requantization kernel of the MP3 decoder, we increase the average number of iterations for this loop from 473 to 576 . This results in $22 \%$ more iterations. The benefit is in the opportunity to merge this kernel with the remaining kernels. Thus we eliminate large intermediate buffers and reduce the number of main memory accesses as we will see in Section 5.

Note, that if the introduced condition is non-innermost, we have to move the if condition to layer 3 . This means locally to repeat step 4.2 for this condition.

\section{RESULTS}

In this section we report our results for all four real-life multimedia applications: a whole MP3 audio decoder, ${ }^{22}$ a requantization and ordering kernel from an audio decoder, a DivX5.X video decoder ${ }^{27}$ and a QSDPCM video coder. ${ }^{34}$ 
We have applied our preprocessing methodology on top of existing preprocessing work as explained in Section 4. After that, the GLT step has been applied (mainly strip mining, loop interchange, and loop fusion) to improve the temporal locality. First strip mining and loop interchange have been applied to be able to fuse the kernels optimally. The script to apply such loop transformations is not the focus of this paper though. A description of the main principles can be found in related work for loop transformations. ${ }^{11,20,26,37}$ After loop fusion, the advanced signal propagation ${ }^{35}$ and in-place ${ }^{17}$ have been used to eliminate large intermediate buffers.

Although beneficial for memory optimisations, the preprocessing introduces usually instruction and controlflow overhead (e.g., code duplication, extending the loop bounds, moving if conditions down in the loop hierarchy and thus execute it more often). However, the postprocessing techniques and tools like the one of Falk et al. ${ }^{13}$ for optimising control flow or code hoisting techniques and common subexpression elimination techniques for elimination of code duplication ${ }^{18}$ can be used to remove the negative preprocessing effects and further improve the code quality.

After these high-level optimisations, we have used the ATOMIUM profiling tool together with Memory Hierarchy Layer Assignment (MHLA) ${ }^{7}$ tool. The MHLA technique performs optimal assignment of arrays and their copy candidates (small data reuse copies to memory closer to the data-path) in the program to the predefined data memory

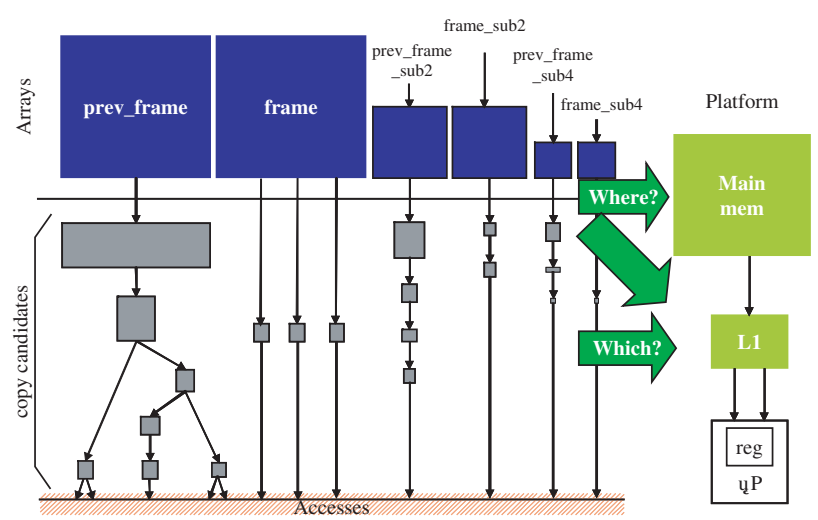

Fig. 7. MHLA mapping decisions (assignment to memory hierarchy) on the QSDPCM application. hierarchy in order to obtain low-power solution (see Fig. 7). We have observed the traffic from/to main memory and we have determined the number of main memory accesses for an optimised memory organisation.

In Table II we can see the results in the number of main memory accesses for our four benchmarks. Column two shows the results for initial applications. Columns three and four show the results for state-of-the-art preprocessed and loop transformed applications. Column five and six show the results for loop transformed applications after both, state-of-the-art and our preprocessing methodology. It is clear that our methodology enables better utilisation of GLT, leading to overall better results when compared to the state-of-the-art approaches without our technique. This will be substantiated in the experiments described below.

We have shown our technique on the MP3 audio decoder through this paper (see Section 3). Using only state-of-the-art work, we are able to optimize $25 \%$ lines of source code of the application, namely frequency inversion and polyphase synthesis filterbank kernels. This leads to $82.2 \%$ reduction in the number of main memory accesses compared to the initial version (see column 4 in Table II). This reduction is mainly due to transformations in the synthesis polyphase filterbank kernel. The remaining kernels cannot be preprocessed and optimised. However, if we apply also our methodology, it enables to optimise $87.5 \%$ lines of source code of the application and leads to a further improvement of $45.8 \%$ (see column 7 and 8 in Table II) in the number of main memory accesses.

A second test vehicle we consider is the requantization and ordering kernels from an audio decoder. The requantization kernel reads each frame in average $400 \times 2 \times 2$ samples. After processing, these samples are written to an intermediate buffer from which they are read into ordering kernel based on data dependent condition. The ordering kernel outputs $576 \times 2 \times 2$ samples. By eliminating the data dependent condition between the requantization and the reordering kernel we are able to merge those two kernels together. This transformation will eliminate the write and read to the intermediate buffer which is quite large and was placed in the off-chip memory by the MHLA tool. It results in the improvement of $35 \%$ in the number of main memory accesses (see column 7 and 8 in Table II) what was not feasible during the presence of the data dependent condition.

Table II. Comparison of \# main memory (MM) accesses of initial application (initial), loop transformed application after existing preprocessing (GLT), and loop transfomred application after our preprocessing for data-dependent constructs on top (PRE+GLT). The last two columns show improvement using our techniques comparing PRE+ GLT and GLT results.

\begin{tabular}{|c|c|c|c|c|c|c|c|}
\hline \multirow[b]{2}{*}{ Application } & \multirow[b]{2}{*}{ Initial \#MM access } & \multicolumn{2}{|c|}{ GLT } & \multicolumn{2}{|c|}{ PRE + GLT } & \multicolumn{2}{|r|}{$\Delta$} \\
\hline & & \#MM acc. & Reduction (\%) & \#MM acc. & Reduction (\%) & \#MM acc. & Reduction (\%) \\
\hline $\operatorname{MP} 3\left[\times 10^{6}\right]$ & 714.2 & 126.9 & 82.2 & 68.8 & 90.4 & 58.1 & 45.8 \\
\hline Req. + Ord. $\left[\times 10^{3}\right]$ & 710.4 & 710.4 & 0 & 460.8 & 35.1 & 249.6 & 35.1 \\
\hline DivX5. $\times\left[\times 10^{6}\right]$ & 9.29 & 9.29 & 0 & 8.98 & 3.3 & 0.31 & 3.3 \\
\hline QSDPCM $\left[\times 10^{3}\right]$ & 542.1 & 445.5 & 17.8 & 306.1 & 43.5 & 139.4 & 31.3 \\
\hline
\end{tabular}


Our third test vehicle is the DivX5.x video decoder. It is extracted from the ffmpeg library of mplayer application ${ }^{27}$ and profiled using dozens realistic DivX movies. This application was already heavily optimised and targeted particularly i686 architecture. Still we have been able to gain 3.3\% (see column 7 and 8 in Table II) in the number of main memory accesses. Without our preprocessing it is infeasible to do any loop transformation on this application. In this application, also a lot of postprocessing (e.g., control-flow optimisation, elimination of code duplication) is needed. This application shows that the benefit of the high-level optimisations can be fully exploited only when targeting the initial specification which is not optimised towards any particular platform.

Finally, the QSDPCM application does not contain all important features for our methodology. It has only one mode resulting in 1 scenario. So the first step of our methodology cannot be applied for this application. Still a lot of data dependent conditions are hidden by applying step 2 of our methodology resulting in $31.3 \%$ gain (see column 7 and 8 in Table II) in the number of main memory accesses compared to existing techniques.

In summary we have created significant additional opportunities for GLT (up to $45.8 \%$ reduction of number of main memory accesses). However, our methodology does not only improve the opportunity for high-level low-power optimisations, particularly GLT. It can also improve the opportunity for low-level optimisations, like the introduction of fine-grain data level parallelism. By enabling loop transformations for our example benchmark, the two channels can be decoded in parallel using SIMD instructions. This can halve the number of remaining main memory accesses and significantly reduce the area and energy cost on top of GLT improvements.

\section{CONCLUSIONS}

Current multimedia programs are characterised as applications with a lot of data dependent constructs. These constructs may considerably hamper optimization opportunities. Therefore, novel preprocessing methodologies are needed.

This paper proposes a systematic preprocessing methodology dealing with data dependent constructs in reallife multimedia applications. The methodology has been explained and demonstrated on the MP3 audio decoder. Finally, results from four real-life multimedia applications have been discussed showing the large potential of this methodology giving reduction in the number of main memory accesses up to $45.8 \%$.

\section{References}

1. J. Absar, F. Catthoor, and K. Das, Call-instance based function inlining for increasing data access related optimisation opportunities. Technical Report, IMEC, Leuven, Belgium (2003).
2. R. Allen and K. Kennedy, Automatic translation of Fortran programs to vector form. ACM Transactions on Programming Languages and Systems (1987), Vol. 9, pp. 491-542.

3. C. Ancourt, D. Barthou, C. Guettier, F. Irigoin, B. Jeannet, J. Jourdan, and J. Mattioli, Automatic data mapping of signal processing applications. Proceedings of the International Conference on Application Specific Array Processors, Zurich, Switzerland (1997), pp. 350-362.

4. D. Atienza et al., Dynamic memory management methodology for reduced memory foot-print in multi-media and wireless network applications. Proceedings of the 7th ACM/IEEE Design and Test in Europe Conf., Paris, France (2004), pp. 532-537.

5. U. Banerjee, R. Eigenmann, A. Nicolau, and D. Padua, Automatic program parallelization. Proceedings of the IEEE, invited paper (1993), Vol. 81, pp. 211-243.

6. C. Bastoul et al., Putting polyhedral loop transformations to work. LCPC'16 International Workshop on Languages and Compilers for Parallel Computers LNCS 2958, College Station (2003), pp. 209-225.

7. E. Brockmeyer, M. Miranda, F. Catthoor, and H. Corporaal, Layer assignment techniques for low energy in multi-layered memory organisations. Proceedings of the 6th ACM/IEEE Design and Test in Europe Conf., Munich, Germany (2003), pp. 1070-1075.

8. F. Catthoor, K. Danckaert, C. Kulkarni, E. Brockmeyer, P. G. Kjeldsberg, T. Van Achteren, and T. Omnes, Data Access and Storage Management for Embedded Programmable Processors, Kluwer Acad. Publ., Boston (2002).

9. M. Cierniak and W. Li, Unifying data and control transformations for distributed shared-memory machines. Proceedings of the SIGPLAN'95 Conference on Programming Language Design and Implementation, La Jolla (1995), pp. 205-217.

10. P. Clauss and V. Loechner, Parametric analysis of polyhedral iteration spaces. J. VLSI Signal Processing, Kluwer (1998), Vol. 19, pp. 179-194.

11. A. Darte and Y. Robert, Affine-by-statement scheduling of uniform and affine loop nests over parametric domains. J. Parallel and Distributed Computing (1995), Vol. 29, pp. 43-59.

12. C. Dezan, H. Le Verge, P. Quinton, and Y. Saouter, The $\{$ Alpha $\}$ du \{CENTAUR $\}$ experiment. Algorithms and Parallel VLSI Architectures II, edited by P. Quinton and Y. Robert, Elsevier, Amsterdam (1992), pp. 325-334.

13. H. Falk and P. Marwedel, Control flow optimization by loop nest splitting at the source code level. Proceedings of the 6th ACM/IEEE Design and Test in Europe Conf., Munich, Germany (2003), pp. 410-415.

14. P. Feautrier, Some efficient solutions to the affine scheduling problems. International Journal of Parallel Programming (1992), Vol. 21, pp. 389-420.

15. A. Fraboulet, G. Huard, and A. Mignotte, Loop alignment for memory access optimization. Proceedings of 12th ACM/IEEE International Symposium on System-Level Synthesis (ISSS), San Jose, CA (1999), pp. 71-70.

16. B. Franke and M. O'Boyle, Array recovery and high-level transformations for DSP applications. ACM Transactions on Embedded Computing Systems (2003), Vol. 2, pp. 132-162.

17. E. De Greef, F. Catthoor, and H. De Man, Program transformation strategies for reduced power and memory size in pseudo-regular multimedia applications. IEEE Trans. on Circuits and Systems for Video Technology (1998), Vol. 8, pp. 719-733.

18. S. Gupta, M. Miranda, F. Catthoor, and R. Gupta, Analysis of highlevel address code transformations for programmable processors. Proceedings of the 3rd ACM/IEEE Design and Test in Europe Conf., Paris, France (2000), pp. 9-13.

19. B. Jung, Y. Jeong, and W. P. Burleson, Distributed control synthesis for data-dependent iterative algorithms. Proceedings on IEEE Int. Conf. on Application Specific Array Processors, San Francisco (1994), pp. 57-68. 
20. M. Kandemir, J. Ramanujam, A. Choudhary, and P. Banerjee, A layout-conscious iteration space transformation technique. IEEE Transactions on Computers (2001), Vol. 50, pp. 1321-1335.

21. W. Kelly and W. Pugh, A framework for unifying reordering transformations. Technical Report CS-TR-3193, Department of CS, University of Maryland, College Park, MD, USA (1993).

22. A. A. Kountouris and $\mathrm{Ch}$. Wolinski, Hierarchical conditional dependency graphs as a unifying design representation in the CODESIS high-level synthesis system. Proceedings of the 13th Intnl. Symposium on System Synthesis, Madrid, Spain (2000), pp. 66-71.

23. K. Lagerström, Design and Implementation of an MP3 Decoder, M.Sc. thesis, Chalmers University of Technology, Sweden (2001), http://www.kmlager.com/mp3/

24. W. Li and K. Pingali, A singular loop transformation framework based on non-singular matrices. Proceedings of 5th Annual Workshop on Languages and Compilers for Parallelism, New Haven, CN (1992).

25. N. Manjiakian and T. Abdelrahman, Fusion of loops for parallelism and locality. Technical Report CSRI-315, Computer Systems Research Institute, University of Toronto, Canada (1995).

26. K. McKinley, S. Carr, and C.-W. Tseng, Improving data locality with loop transformations. ACM Trans. on Programming Languages and Systems (1996), Vol. 18, pp. 424-453.

27. http://www.mplayerhq.hu/

28. R. Olsen and G. Gao, Collective analysis and transformation of loop clusters. Technical Report, ACAPS Technical Memo 24, McGill University (1992).

29. M. Palkovic, E. Brockmeyer, and F. Catthoor, Hierarchical rewriting and hiding of data dependent conditions to enable global loop transformations. Proceedings of 2nd Workshop on Optimisation for DSP and Embedded Systems, Palo Alta, CA (2004).

30. M. Palkovic, H. Corporaal, and F. Catthoor, Global memory optimisation for embedded systems allowed by code duplication. Proceedings of 9th International Workshop on Software and Compilers for Embedded Systems, Dallas, TX (2005).

31. C. Polychronopoulos, Compiler optimizations for enhancing parallelism and their impact on the architecture design. IEEE Transactions on Computers (1988), Vol. 37, pp. 991-1004.

32. W. Pugh, The omega test: A fast and practical integer programming algorithm for dependence analysis. Communications of the ACM (1992), Vol. 35.

33. L. Semeria and G. De Micheli, SpC: Synthesis of pointers in C. Proceedings of IEEE International Conference on Comp. Aided Design, Santa Clara, CA (1998), pp. 340-346.

34. $P$. Strobach, QSDPCM-A New technique in scene adaptive coding. Proceedings of the 4th European Signal Processing Conference, Grenoble, France (1988), pp. 1141-1144.

35. P. Vanbroekhoven, G. Janssens, M. Bruynooghe, H. Corporaal, and F. Catthoor, Advanced copy propagation for arrays. Proceedings of the SIGPLAN Conference on Languages, Compilers, and Tools for Embedded Systems (LCTES'03), San Diego, CA (2003), pp. 24-33.

36. D. Wilde, A Library for doing Polyhedral Operations, M.Sc. thesis, Oregon State University, in co-operation with IRISA/INRIA, Rennes, France (1993).

37. M. Wolf and M. Lam, A data locality optimizing algorithm. Proceedings of the SIGPLAN'91 Conference on Programming Language Design and Implementation, Toronto, Canada (1991), pp. 30-43.

\section{Martin Palkovic}

Martin Palkovic received his B.Sc. and M.Sc. degrees in Electrical Engineering from the Slovak University of Technology in Bratislava, Slovakia in 1999 and 2001. Since 2001 he is a researcher at the Interuniversity Micro-Electronics Center (IMEC) in Heverlee, Belgium. His research interests include high-level optimisations in data dominated multimedia applications, related system design automation aspects, and platform architectures for low power.

\section{Erik Brockmeyer}

Erik Brockmeyer received his M.Sc. degree in Electrical Engineering from the Technische Universiteit Eindhoven, The Netherlands in 1998. Since 1998 he is a researcher at the Interuniversity Micro-Electronics Center (IMEC) in Heverlee, Belgium. He has worked on automation of various steps of the Data Transfer and Storage Exploration (DTSE) script, particularly on mapping an application efficiently to the multi levels of a memory hierarchy. Currently, his research work is shifting toward multi-processor systems with a shared distributed memory subsystem.

\section{Peter Vanbroekhoven}

Peter Vanbroekhoven received his B.Sc. and M.Sc. degrees in Computer Science from the Katholieke Universiteit Leuven (KU Leuven), Belgium in 1997 and 2000, respectively. Since 2000 he is a researcher at the Katholieke Universiteit Leuven in Belgium. His research interests include compiler techniques in general and high-level optimizations in data-dominated multimedia applications in particular.

\section{Francky Catthoor}

Francky Catthoor received the engineering degree and the Ph.D. degree in Electrical Engineering from the Katholieke Universiteit Leuven (KU Leuven), Belgium in 1982 and 1987, respectively. Since 1987, he has headed several research domains in the area of highlevel and system synthesis techniques and architectural methodologies, all within the Design Technology for Integrated Information and Telecom Systems (DESICS_formerly VSDM) division at the Interuniversity Micro-Electronics Center (IMEC), Heverlee, Belgium. Currently, he is an IMEC fellow. He is a part-time full professor in the Electrical Engineering Department at KU Leuven. In 1986, he received the Young Scientist Award from the Marconi International Fellowship Council. He has been an associate editor for several IEEE and ACM journals, such as the IEEE Transactions on VLSI Signal Processing, IEEE Transactions on Multimedia, and ACM Transactions on Design Automation of Electronic Systems. He was the program chair of several conferences, including ISSS '97 and SIPS '01. He is a fellow of the IEEE and a member of the IEEE Computer Society.

\section{Henk Corporaal}

Henk Corporaal received the M.Sc. degree in theoretical physics from the University of Groningen and the Ph.D. degree in Electrical Engineering (in the area of computer architecture) from Delft University of Technology. Currently, he is a professor of embedded 
system architectures at the Einhoven University of Technology (TU/e) in The Netherlands and director of research of DTI, the joint Design Technology Institute of TU/e and NUS (National University of Singapore). Previously he taught at several schools for higher education, worked at the Delft University of Technology in the field of computer architecture and code generation, and has been department head and chief scientist within the DESICS (Design Technology for Integrated Information and Communication Systems) division at IMEC, Leuven, Belgium. He has coauthored many papers in the processor architecture and design area and written a book on a new class of VLIW architectures, the Transport Triggered Architectures. 